\title{
Wireless Nano Senor Network (WNSN) for Trace Detection of Explosives: The Case of RDX and TNT
}

\author{
Rejeti Venkata Kishore Kumar ${ }^{1 *}$, Gonavath Manthru Naik², Gudipati Murali ${ }^{1}$ \\ ${ }^{1}$ Department of Computer Science and Engineering, KKR \& KSR Institute of Technology and Sciences, Guntur 522017, AP, \\ India \\ ${ }^{2}$ Department of Computer Science and Engineering, Sri Mittapalli Institute of Technology for Women, Guntur 522019, AP, \\ India
}

Corresponding Author Email: mail2rvkk@gmail.com

https://doi.org/10.18280/i2m.180209

Received: 12 January 2019

Accepted: 6 April 2019

\section{Keywords:}

Nano sensor, WNSN, trace explosive detection, terrorism, TNT, RDX

\begin{abstract}
Due to enormous growth in explosives-based terrorism across the globe, the research on detection of explosives assumed unprecedented significance. It is more so in the wake of 9/11 and more recent incidents. There is no single solution for the menace of terrorism. One of the solutions is to detect explosives early and build counterintelligence to combat the man-made campaign. However, it is a challenging task to detect trace explosives due to low vapour pressure, increasing permutations in composition, avenues for deployment, and concealment. Many trace explosive detection systems came into existence. They include sensor based detection, Gas Chromatography (GC), Mass Spectrometry (MS), and Mobility Spectrometry (MS). These techniques need devices that are expensive, bulky and with operating procedures that are time taking. Deploying such devices in strategic areas is inadequate as the large scale usage of them is not possible. Therefore it is indispensable to have small inexpensive sensors, which are sensitive and selective, deployed in large scale. Nano sensor technology when leveraged to form a massive network has potential for detecting trace explosives. As a step toward the solution, we presented a methodology with Wireless Nano Sensor Network (WNSN) implemented using NS2 for simulation study of trace detection of explosives. We proposed an algorithm named Nano Trace Explosive Detection (NTED) to achieve this. As there was a plethora of explosives materials, we preferred the two most common explosives named TNT and RDX as case study. Our results revealed the significance and potential of WNSN for protecting people and properties from terror attacks.
\end{abstract}

\section{INTRODUCTION}

Nano sensors are becoming potential platform for various applications that are distributed in nature including explosive detection. Especially nanochecmical sensors came into existence for that can achieve high selectivity and sensitivity. Moreover nonosensors support both receptor-free and receptor-based approaches and can fit well into sensor networks. Since explosive based terrorism is growing rapidly in recent years, the research on explosive detection assumed significance. However, it is very challenging to have large scale surveillance for explosive detection. There are many factors that make it a challenging task. They include novel materials introduced for explosives, most explosives containing low vapour pressure, different novel permutations of explosives, and weapon delivery and concealment schemes. There are two kinds of detection mechanisms for explosives. They are known as trace explosive detection and bulk explosive detection. Most important techniques available for trace explosives include sensor based detection, Gas Chromatography (GC), Mass Spectrometry (MS), and Mobility Spectrometry (MS).

The problem with above mentioned approaches needs huge devices that are not affordable for large scale deployment of sensors in the wake of ever-increasing terror attacks prevailing in different parts of the world. Therefore it is important to have affordable solutions. In this paper, we focused on trace explosive detection methodology. Trace detection involves collection of vapour and analyzing it using sensors. Fast detection of explosives and provision continuous operation are important for monitoring explosives with real time detection and notification. Therefore mass deployment of sensors is important. Towards this end, the traditional equipment is not viable and not feasible as well. Therefore, it is the time to have more convenient and yet cost-effective approach for large scale sensor networks deployed in large geographical area. With advent of nanoscience associated with sensors, things are gradually changing. This is the rationale behind choosing nanosensor network for explosive detection in this paper.

Our contributions in this paper are as follows. We proposed an algorithm named Nano Trace Explosive Detection (NTED) for trace explosive detection. We considered TNT and RDX traces for experiments. We proposed a system model and implemented it with simulations to demonstrate proof of the concept. The results revealed that the proposed algorithm is effective and able to detect trace explosives with high precision. The remainder of the paper is structured as follows. Section 2 provides review of literature on detection of explosives and related aspects. Section 3 presents bulk and trace detection methods and nano sensors. Section 4 presents 
the properties of explosives such as RDX and TNT as they are considered as case study in this paper. Section 5 presents the proposed methodology for detection of trace explosives. Section 6 presents experimental evaluation. Section 7 concludes the paper besides providing directions for future work.

\section{RELATED WORKS}

This section provides review of literature on different ways detecting explosives in the real world. Mohan and Shelly [1] studied explosive detection in border areas. Since border areas need to handle threats from people and their anti-terror activities, they explored border security robot with two PIR sensors for detecting person and detecting explosives respectively. They studied different technologies involved in the system. They include Bluetooth technology and infrared technology. They implemented a simulation study in Visual Basic. Ball et al. [2] studied military surveillance networks and the usage of computational intelligence for explosive detection. Kumar and Murali [3] made a review of explosives, their detection methods and possible automatic detection of explosives using Wireless Sensor Network (WSN). Kumar and Sushanth [4] explored robotic vehicle with an arm for explosive detection. The arm is able to understand the presence of poisonous gas in the air. Makeenkov et al. [5] on the other hand studied infrared sensor nodes for monitor liquids for their explosive behaviours. It can sense the lower explosive limit of gases.

Usmanov et al. [6] performed analysis of explosives with a method known as alternating current corona discharge. It find source of atmospheric pressure chemical ionization by exploiting density functional theory computations. They studied the explosive behaviour of TNT, RDX and other explosives. Lopez et al. [7] focused on the problem of explosion of gas cylinders. Gas cylinders are widely used for cooking gas. In this context, various incidents pertaining to explosion of cylinders were witnessed from time to time. They made analysis of such incidents and made empirical study of natural gas and high-pressure hydrogen gas cylinders. They employed TNT equivalent method for detecting explosives. Gas detection can also help in understanding explosives and detection. Towards this end, Rehman and Zeng [8] studied ionic liquids as gas materials for gas sensing. They found that ionic liquids do have sensing capabilities that can be exploited for the purpose of explosive detection. Millar et al. [9] studied RDX and its characteristics in terms of its crystal structure. Various distraction techniques are employed for characterization of RDX in terms of its structure.

Shen et al. [10] employed spectroscopy imaging for identification and detection of explosives. The imaging is used at terahertz pulse range. In fact they combined both chemical mapping of explosives and terahertz spectroscopy for effective detection of explosives. Tiwari and Premi [11] focused on highly flammable gases use in a factory. They performed vulnerability analysis to understand the possibility of fire or explosion. In order to achieve this, they employed a method known as Vapour Cloud Explosion Modelling (VCEM). Here the vulnerability is the ease of making an attack on it for malicious or terror activities. Millar et al. [12] studied a variant of RDX to understand various factors related to detonation. They include sensitivity to detonation, detonation velocity, and crystal density. Sarabia et al. [13] focused on nitro explosive vapours in order to achieve selective detection and discrimination. For this, they used three flurogenic sensory polymer membranes. For discrimination purposes, they employed Principal Component Analysis (PCA). A measure known as Soft Independent Modelling of Class Analogy (SIMCA) is used for finding the difference among the three explosives.

Watson and McGuire [14] used Gas Chromatography (GC) along with an electronic nose and Surface Acoustic Wave (SAW) for detection of explosives that are in the water and soil. They performed analysis of the materials to have quantifiable response for determination of the kind of explosives. They employed Surface Acoustic Wave (SAW) detector along with GC for faster recognition of the presence of explosives in water and soil. Vanimireddy and Kumari proposed a detection method for Improvised Explosive Devices (IED) that is generally deployed in public places by terrorists. They proposed architecture with different sensors to detect explosive materials. Rebuffel et al. [15] studied the semi-conductor based explosive detection mechanisms and evaluate explosive detection system (EDS). They made empirical study to understand the behaviour and characteristics of explosive materials based on spectrometric detectors

\section{EXPLOSIVE DETECTION METHODS AND NANOSENSORS}

Explosive detection methods are broadly classified into bulk and trace explosive detection methods. Burn rates of explosives are considered to understand them as high intensity or low intensity explosives. These explosives exhibit selfdecay propagation in order to release heat and pressure while causing explosion. As the name implies, bulk explosives are the explosives that are made up of many materials and they are used in large scale directly for explosion activities. A detection method used for bulk explosives is known as bulk explosive detection methods. On the other hand trace explosives are the explosives that are used in small magnitude. They are detected by trace explosive detection methods.

Bulk detection methods are divided into sixcategories. They are X-rays, neutrons, electromagnetic imaging, electromagnetic and Y rays. X-rays based approaches based on transmission, dust energy, backscatter, $\mathrm{x}$-ray with high flux sources and remote detectors. Computer tomography also comes under this category. The Neutrons approaches include thermal neutron analysis, fast neutron analysis, pulsed fast neutron analysis, pulsed fast thermal neutron analysis, etc. Electromagnetic imaging approaches include radar, microwave, terahertz, and infrared. Electromagnetic approaches are magnetic field, ESR, NMR and NQR while Y rays approaches include backscatter and transmission. These methods are used to detect bulk explosives. On the other hand trace explosive detection methods detect explosive based on the traces or residues of explosive materials. Figure 2 shows trace explosive detection methods.

Trace detection method are broadly classified into three types. They are chemical or electronic, optical and biosensors based approaches. Biosensors are made up of living things and even robotics. Some of the widely used trace explosive detection methods are sensor based detection, Gas Chromatography (GC), Mass Spectrometry (MS), and Mobility Spectrometry (MS). Trace explosive methods 
depend on either vapour or particulate samples. Different vapours pertaining to RDX, TNT and other explosive materials include nitroaromatics, nitroaminies, nitroesters, acid salt, ammonium picrate, and organic peroxides. RDX is associated with the vapour nitroamines while the TNT is associated with nitroaromatics. Nitroamines for vapour trace explosive detection are associated with the explosives such a RDX: 1, 3, 5-triazacyclohexane, HMX: Octahydro- 1,3,5,7 tetranitro- 1,3,5,7 -tetrazocine and NQ: Nitro guanidine. Explosives associated with nitroaromatics include TNT: 2,4,6Trinitrotoluene, TNB: 1,3,5- Trinitrobenzene, DNB: 1,3Dinitrobenzene, 2, 4 DNT: 2,4 - Dinitrotoluene, 2, 6-DNT: 2, 6 -dinitrotoluene, Tetryl: Methyl-2, 4, 6-trinitrophenylnitramine, 2AmDNT: 2-amino-4, 6 - dinitrotoluene, 4AmDNT: 4-amino-2, 6 - dinitrotoluene, NT: Nitrotoluene (3 isomers), NB: Nitrobenzene and EGDN: Ethylene glycol dinitrate. Ammonium nitrate and urea nitrate are related to acid salt. Nitroesters are associated with NG: Nitroglycerin (glycerol trinitrate) and PETN: Pentaerythritol tetra nitrate. Picric acid relted exlsoive materials are AP/PA: Ammonium 2, 4, 6-trinitrophenoxide/2, 4, 6- trinitrophenol while organic peroxides are TATP: Triacetone tripede and HMTD: Hexamethylene triperoxide diamine. Out of these trace explosive detection methods, many can be used in nanotechnologies based approaches. They include electrochemical, photoluminescence, mass-based, fibre-optic based, IMS, laserinduced breakdown spectroscopy, surface enhanced raman scattering, tera hertz detection, and biosensors.
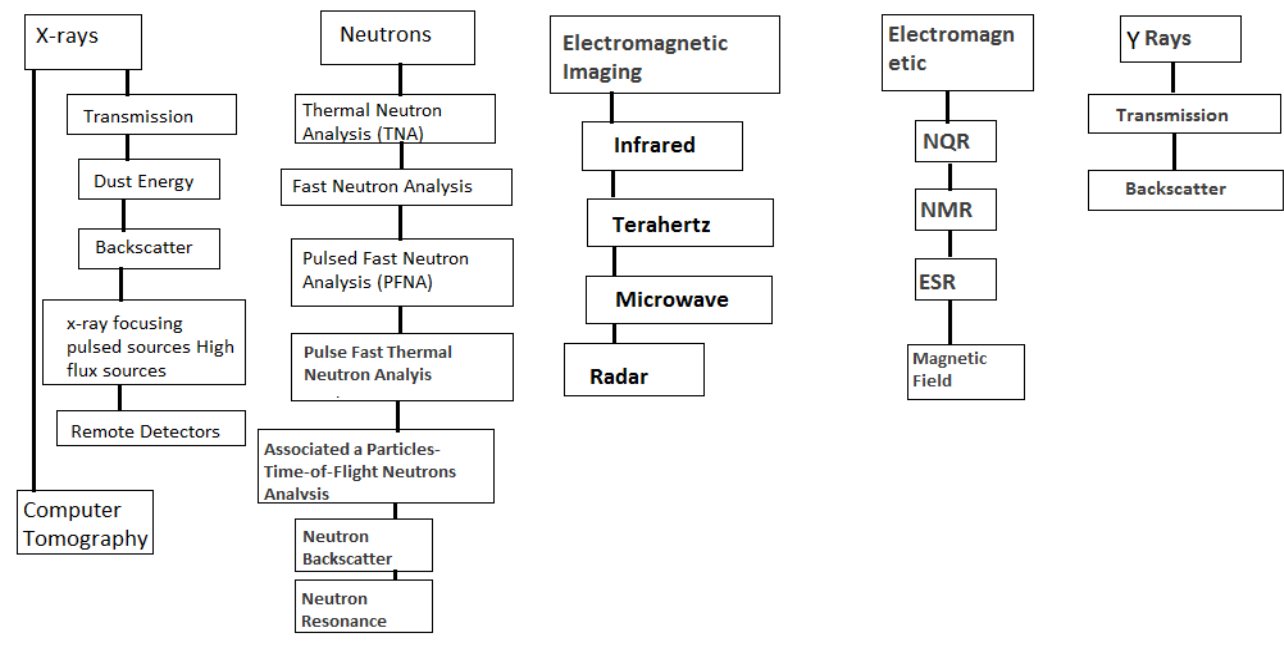

Figure 1. Bulk explosion detection methods

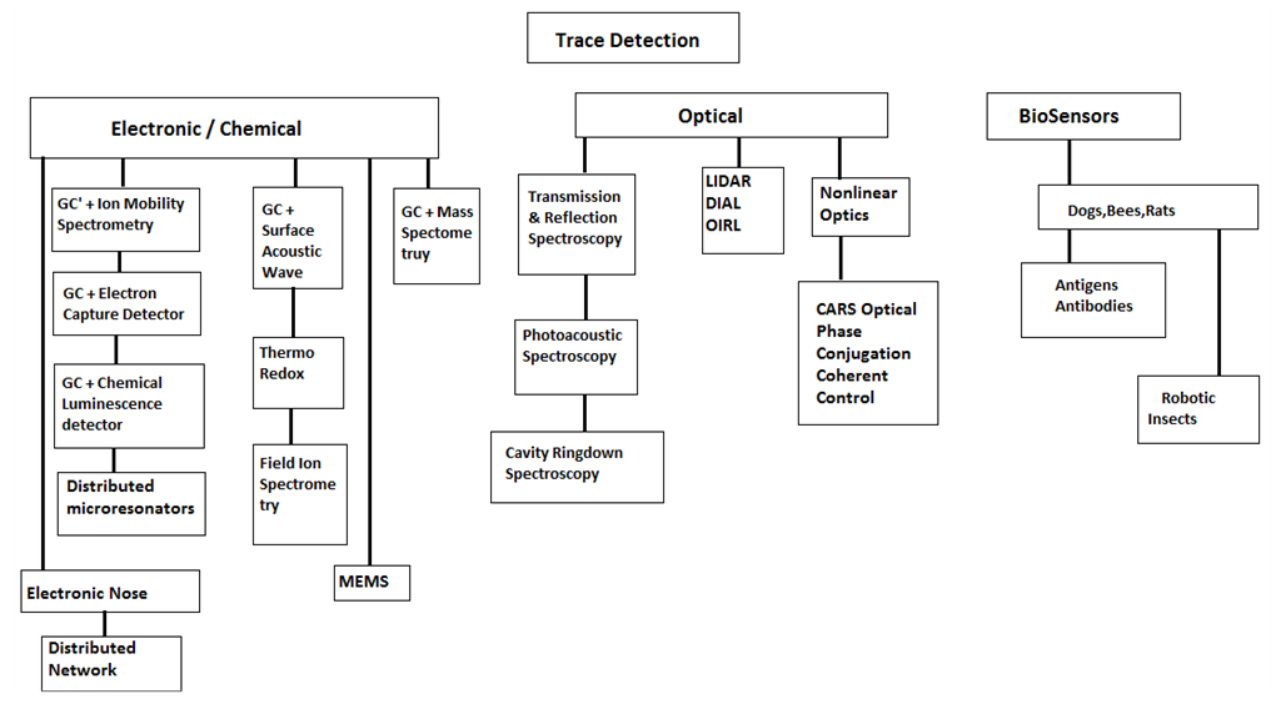

Figure 2. Trace explosive detection methods

\section{METHODOLOGY FOR DETECTION OF TRACE EXPLOSIVES}

In this paper, we focused on nanosensor based approach for explosive detection. The rationale behind this is that nanosensors are very small with enabled sensor capabilitiesfor diversified purposes. It is suitable for large scale deployment so as to combat the menace of terrorism. First of all the traces of TNT and RDX are collected. As mentioned above, trace explosive detection is very challenging as getting traces is a tedious task. The rationale behind this is that many explosives release vapour which has very low pressure. That is the reason huge amount of air with traces needs to be collected for efficient experimentation. Thus it is possible to have enough molecules of explosives for experiments. Again it is base on the sensitivity of nanosensors. When nanosensor is highly 
sensitive, it is possible to do experiments with less air containing traces of explosives. Otherwise more molecules are required. It is understood that the sensitivity of sensors is not adequate. That is the reason it is important to collected more molecules for experiments. Large volume of air needs to be considered for containing sufficient molecules related to the explosive in question.

The pre-concentrators that are conventional are bulky in nature. They need more space, more power, and exhibit low response time. Therefore they are not suitable for large scale deployment of sensors for real time detection of explosives. When it comes to nanosensors, they are very handy, small in size, have ability to have high efficiency when compared with their predecessors with respect to sampling. Recent innovations reveal the utility of nanosensor devices to form a sensing network that can be deployed in large scale. The devices are highly useful for such deployment as needed. With sorbent material used in USA recently, faster desorption of explosive molecules is possible.

In is understood from the literature that nano technology related to sensors can provide unprecedented possibilities in the research of trace detection of explosives. The nano scale features can influence chemical and physical properties of sensors positively. And the technology enables sensors to have real time sensing capabilities besides making the deployment of huge number of sensors in large scale feasible and affordable. Moreover, nano technology enabled equipment can help in working in tandem with sensor devices. As the sensor's dimension is smaller, it is able to help in increase of sensitivity. At the same time, it can achieve more capabilities in sensing chemicals. Nevertheless, many nanosensors in the real world exhibit pool selectivity. Though the technology is in its infancy, it has created an impression that it is going to prevail in the explosive detection mechanisms across the globe in future. Optical, mechanical, physical and magnetic properties are enhanced with nanoscale effects. With nano technologies, it is possible to have bottom up approaches considering atoms and molecules for analysis and building a method for trace explosive detection. Nanomechanical effects with molecular-absorption make nanosensors good candidates for trace explosive detection. In this paper we propose a system model for Nanosensor Wireless Network (NSWN) which is meant for detection of trace explosives. The architectural overview of the system model is shown in Figure 3.

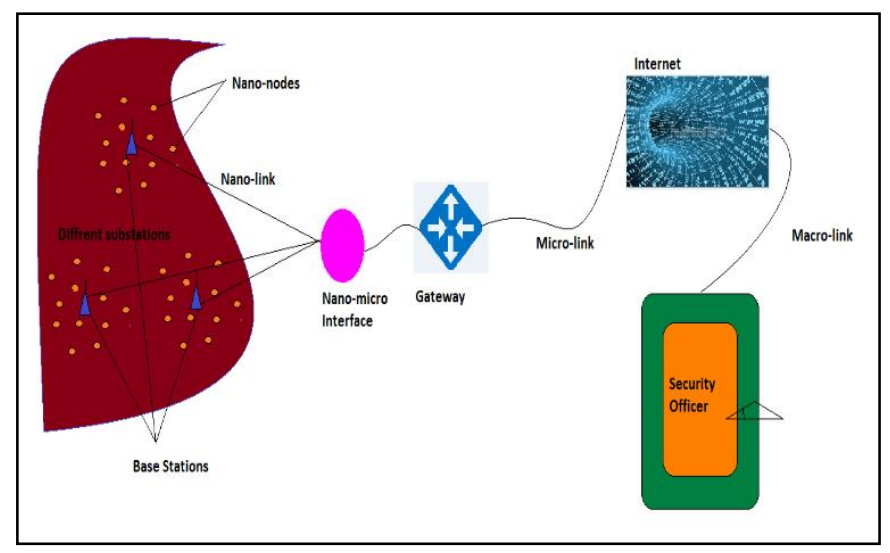

Figure 3. System model

Nanosensor networks are spread geographically since they are supposed to cover large area which needs to provide surveillance for explosive detection. Multiple wireless nanosensor networks are integrated with Internet so as to enable users to gain access to the data produced by them. A security officer can make queries and obtain information needed. Many nano-sensor nodes are part of a network. Each node is capable of sensing residues of TNT and RDX which are in the air. The molecules of these materials are produced in simulated environment in order to demonstrate trace explosive detection. Nanosensors in each network are connected to a base station. From each base station, nano links are established to nano-micro interface. Through nano micro interface all base stations are connected to a gateway. The gateway is connected to Internet and thus data is stored in an Internet repository which is accessed by authorized security personnel.

\subsection{Nano trace explosive detection algorithms}

This algorithm is defined to work at each edge for real time edge analytics so as to speed up notification of trace detection of explosives. This algorithm analyzes simulated values associated with TNT and RDX and then provides detection results. The algorithm runs in each sensor node so as to enable each one to have capability of sensing explosive materials.

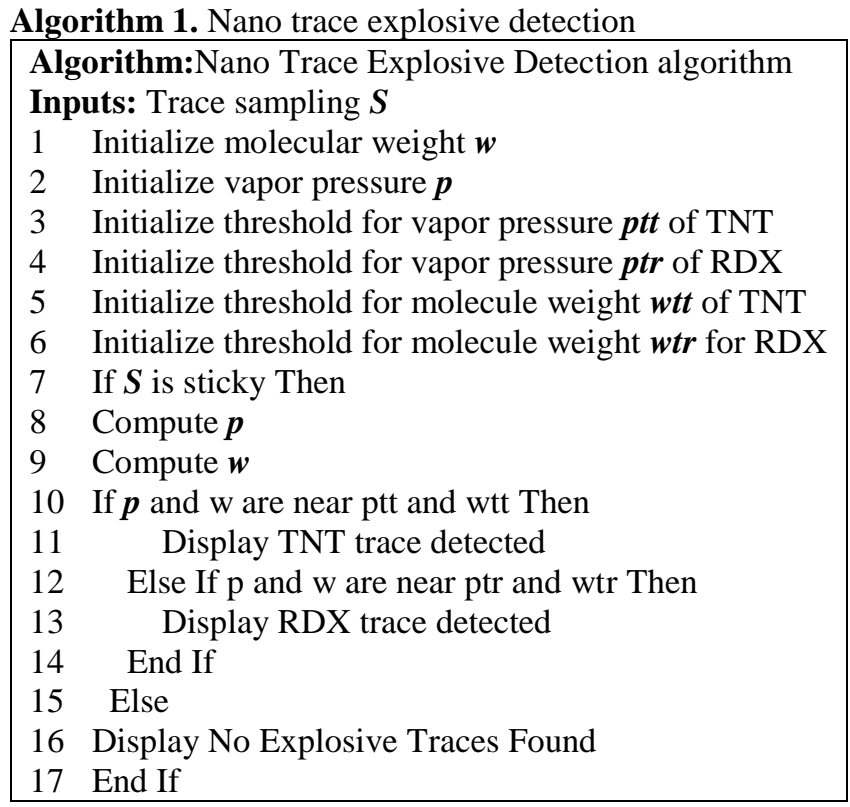

As presented in Algorithm 1, the trace explosive detection process includes sampling of explosive trace which is subjected to detection of explosives. Two explosives such as TNT and RDX that are commonly used are considered for experiments. The ground truth values provided in Table 1 are used for experiments with simulations.

\section{EXPERIMENTAL RESULTS}

Experiments are made with NS2 simulation environment for demonstrating trace explosive detection. The system model presented in this paper is used to perform experiments. The characteristics of explosives such as TNT and RDX are used as described in Table 1 for trace detection of explosives. Precision and recall measures are computed for evaluation of the proposed method. The experiments are made 100 times. The observations are recorded. The precision and recall are 
computed based on the confusion matrix presented in Table 1.

Table 1. Confusion matrix

\begin{tabular}{|c|c|c|}
\hline & $\begin{array}{l}\text { Ground Truth } \\
\text { (correct trace } \\
\text { explosive } \\
\text { detection) }\end{array}$ & $\begin{array}{c}\text { Ground Truth } \\
\text { (incorrect trace } \\
\text { explosive decision) }\end{array}$ \\
\hline $\begin{array}{l}\text { Algorithm (correct } \\
\text { trace explosive } \\
\text { detection) }\end{array}$ & $\begin{array}{c}\text { True Positive } \\
\text { (TP) }\end{array}$ & $\begin{array}{l}\text { False Positive } \\
\text { (FP) }\end{array}$ \\
\hline $\begin{array}{c}\text { Algorithm } \\
\text { (incorrect trace } \\
\text { explosive } \\
\text { detection) } \\
\end{array}$ & $\begin{array}{l}\text { False Negative } \\
\quad(\mathrm{FN})\end{array}$ & $\begin{array}{l}\text { True Negative } \\
\text { (TN) }\end{array}$ \\
\hline
\end{tabular}

Confusion matrix is used to as the basis for evaluation of the proposed algorithm Nano Trace Explosive Detection. Precision and recall are statistical measures that are based on the confusion matrix. They are computed as in Eq. (1) and Eq. (2).

$$
\text { Precision }=\mathrm{TP} /(\mathrm{TP}+\mathrm{FP})
$$

$$
\text { Recall }=\mathrm{TP} /(\mathrm{TP}+\mathrm{FN})
$$

Precision refers to the ratio of correctly detected trace explosive experiments to the total number of experiments made. In the same fashion, recall is the ratio of number of actual correctly detected trace explosive experiments out of correctly detected trace explosive experiments.

Table 2. Shows precision and recall values

\begin{tabular}{ccc}
\hline TP & FP & FN \\
\hline 0.002075 & 1 & 0.22825 \\
0.197095 & 0.95 & 0.22935 \\
0.373444 & 0.904523 & 0.22989 \\
0.53112 & 0.85906 & 0.23053 \\
0.653527 & 0.793451 & 0.23142 \\
0.755187 & 0.733871 & 0.23196 \\
0.838174 & 0.678992 & 0.23259 \\
0.906639 & 0.628777 & 0.23301 \\
0.962656 & 0.583648 & 0.23368 \\
0.995851 & 0.536313 & 0.23411 \\
1 & 0.482 & 0.23486 \\
\hline
\end{tabular}

As shown in Table 2, it is evident that there the values required by the measures precision and recall are provided for some select experiments. In fact the Table 3 shows an excerpt from the results obtained.

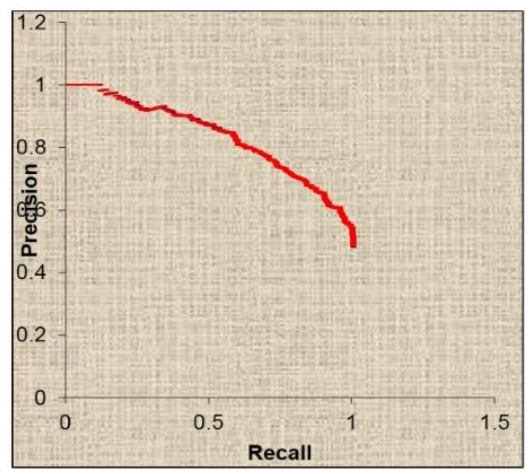

Figure 4. Precision and recall evaluation
As can be seen in Figure 4, it is evident that recall is represented in horizontal axis and precision is represented by vertical axis. The results reveal the performance of the proposed simulated trace explosive detection method. Each measure is 0.0 to 1.0 showing the ratio to understand the quality of the trace explosive detection method.

\section{CONCLUSIONS AND FUTURE WORK}

Many techniques came into existence as found in the literature for both bulk and trace detection of explosives. However, it is understood that there is no single solution that is capable of fulfilling objectives of anti-terrorism. In this paper, we studied trace detection of explosives using Wireless Nano Sensor Network (WNSN). Due to the emergence of nano technologies and the capabilities of them to serve for different sensing purposes, they are good candidates for trace explosive detection. Expensive and bulky devices for explosive detection are no longer suitable as terrorism can occur at any corner of the world and large scale usage of such devices is not feasible. Therefore it is inevitable to have small devices whose presence cannot be detected easily for sensing explosives and provide real time feedback to the people concerned. A massive sensor network possibly made up of thousands of nano sensors seems to have an attractive solution for detection of explosives. In this paper, therefore, we investigated the utility of nano sensors for trace explosive detection. We considered the case of two explosives that are widely used. They are RDX and TNT traces that are sensed by nanosensors. Towards this end we proposed an algorithm named Nano Trace Explosive Detection (NTED) that utilizes characteristics of explosive traces and detect the presence of RDX or TNT and provide notifications. Our simulation results revealed that the proposed algorithm is effective in detecting trace explosives. In future, we intend to make experiments with other kinds of explosives besides improving algorithms for trace explosive detection.

\section{REFERENCES}

[1] Mohan, M., Shelly, S. (2016). Border security robot. International Journal on Cybernetics \& Informatics, 5(2): 275-283. https://doi.org/10.5121/ijci.2016.5230

[2] Mark, G., Ball, B., Wesolkowski, S. (2015). A review of the use of computational intelligence in the design of military surveillance networks. Recent Advances in Computational Intelligence in Defense and Security, 663-693. https://doi.org/10.1007/978-3-319-26450-9 24

[3] Kumar, R.V.K., Murali, G. (2016). An accurate methodology to identify the explosives using wireless sensor networks. Proceedings of International Conference on Sustainable Computing in Science, Technology and Management (SUSCOM), Amity University Rajasthan, Jaipur, India, https://doi.org/10.2139/ssrn. 3362178

[4] Kumar, N., Sushanth, K.J. (2016). Gesture contolled robotic arm using accelerometer. International Journal of Core Engineering \& Management (IJCEM), 3(1): 91-101.

[5] Makeenkova, A., Lapitskiya, I., Somov, A., Baranovca, A.A. (2015). Flammable gases and vapors of flammable liquids: Monitoring withinfrared sensor node. Sensors and Actuators B: Chemical, 209: 1102-1107. https://doi.org/10.1016/j.snb.2014.11.112 
[6] Tsmanov, D.T., Chen, L.C., Yu, Z., Yamabe, S., Sakakic, S., Hiraoka, K. (2015). Atmospheric pressure chemical ionization of explosives using alternating current corona discharge ion source. Journal of Mass Spectrometry, 651-661. https://doi.org/10.1002/JMS.3552

[7] López, E., Rengel, R., Mair, G.W., Isorna, F. (2015). Analysis of high-pressure hydrogen and natural gas cylinders explosions through TNT equivalent method. Conference: V Iberian Symposium on Hydrogen, Fuel Cells and Advanced Batteries (HYCELTEC), At Tenerife, Spain, 35-42 https://doi.org/10.13140/RG.2.1.2336.8401

[8] Rehman, A., Zeng, X.Q. (2015). Methods and approaches of utilizing ionic liquids as gas sensing materials. The Royal Society of Chemistry, 5(72): 58371-58392. https://doi.org/10.1039/C5RA06754E

[9] David, I.A., Millar, I., Oswald, D.H., Barry, C., Francis, D.J., Marshall, W.G., Pulham, C.R., Cumming, A.S. (2017). Pressure-cooking of explosives--the crystal structure of epsilon- RDX as determined by X-ray and neutron diffraction. Chemical Communications, 46(31): 5662-5664. https://doi.org/10.1039/c0cc00368a

[10] Shen, Y.C., Lo, T., Taday, P.F., Cole, B.E., Tribe, W.R., Kemp, M.C., (2005). Detection and identification of explosives using terahertz pulsed spectroscopic imaging.
Applied Physics Letters, 86(24): 1-4. https://doi.org/10.1063/1.1946192

[11] Tiwari, A., Premi, R. (2016). Vulnerability analysis of a factory using highly flammable gases. IJSRSET, 2(2): 910-914.

[12] Millar, D.I., Oswald, I.D., Francis, D.J., Marshall, W.G., Pulham, C.R., Cumming, A.S. (2017). The crystal structure of beta-RDX-an elusive form of an explosive revealed. Chemical Communications, 5: 562-564. https://doi.org/10.1039/B817966B

[13] Pablos, J.L., Sarabia, L.A., Ortiz, M.C., Mendía, A., Muñoz, A., Serna, F., García, F.C., García, J.M. (2015). Selective detection and discrimination of nitro explosive vapors using an array of three luminescent sensory solid organic and hybrid polymer membranes. Sensors and Actuators B: Chemical, 212: 18-27. https://doi.org/10.1016/j.snb.2015.01.103

[14] Yinon, J., McGuire, D.S. (2003). Detection of explosives by electronic nose. Analytical Chemistry, 75(5): 98-105. https://doi.org/10.1021/ac0312460

[15] Loick, V., Eric, G., Olivier, M., Guillaume, M., Bernard, P. (2007). New trends in $\gamma$-ray imaging with $\mathrm{CdZnTe} / \mathrm{CdTe}$ at CEA-Leti. Nuclear Instruments and Methods in Physics Research Section A, 571(1-2): 33-43. https://doi.org/10.1016/j.nima.2006.10.023 